\title{
L'APPARITION DE LA MONNAIE FRAPPÉE : INVENTION OU INNOVATION ? BILAN HISTORIOGRAPHIQUE ET ENJEUX HISTORIQUES
}

\author{
Pierre-Olivier Hochard
}

Presses Universitaires de Franche-Comté | « Dialogues d'histoire ancienne »

2020/Supplement20 S 20 | pages 15 à 34

ISSN 0755-7256

Article disponible en ligne à l'adresse :

https://www.cairn.info/revue-dialogues-d-histoireancienne-2020-Supplement20-page-15.htm

Distribution électronique Cairn.info pour Presses Universitaires de Franche-Comté.

(C) Presses Universitaires de Franche-Comté. Tous droits réservés pour tous pays.

La reproduction ou représentation de cet article, notamment par photocopie, n'est autorisée que dans les limites des conditions générales d'utilisation du site ou, le cas échéant, des conditions générales de la licence souscrite par votre établissement. Toute autre reproduction ou représentation, en tout ou partie, sous quelque forme et de quelque manière que ce soit, est interdite sauf accord préalable et écrit de l'éditeur, en dehors des cas prévus par la législation en vigueur en France. Il est précisé que son stockage dans une base de données est également interdit. 


$$
\text { - I - }
$$

LES DÉbUtS DE LA MONNAIE ANTIQUe 


\title{
L'APPARITION DE LA MONNAIE FRAPPÉE : INVENTION OU INNOVATION? BILAN HISTORIOGRAPHIQUE ET ENJEUX HISTORIQUES
}

\author{
Pierre-Olivier Hochard \\ Université de Tours - CeTHiS (EA 6298) \\ pierre-olivier.hochard@univ-tours.fr
}

Il semble aujourd'hui difficile d'envisager notre vie quotidienne sans le recours à la monnaie. Encore faut-il s'entendre sur la signification de ce mot. Si l'on se fie à la première définition du terme donnée par le dictionnaire Larousse 2017, la monnaie est une « pièce de métal frappée par l'autorité souveraine pour servir aux échanges ». Cette définition rejoint en partie celle donnée par les auteurs de La monnaie grecque dans leur introduction : la monnaie est généralement définie comme « un morceau de métal dont l'estampille garantit à la fois le poids et le titre $»^{1}$. Ces définitions ne vont cependant pas sans poser quelques difficultés. L'idée d'une pièce ou d'un morceau de métal n'a en effet rien d'évident. J.-J. Rousseau dans son Émile (livre III) relevait que :

tout peut être monnaie; autrefois le bétail l'était, des coquillages le sont encore chez plusieurs peuples, le fer fut monnaie à Sparte, le cuir l'a été en Suède, l'or et l'argent le sont parmi nous.

Comme le soulignait ainsi C. Grandjean dans l'introduction à la journée d'études Pré, paléo, proto, para, péri, qu'est-ce que la monnaie?:

L'étude des monnayages d'autres régions du globe révèle l'existence d'instruments monétaires très différents par leur forme, leurs matières et leurs fonctions auxquels est très souvent refusée la qualité de monnaie [et] dont l'utilisation n'est pas toujours uniquement monétaire (pains de sel, pièces d'étoffes, couteaux, perles, etc.) et/ou qui coexistent avec d'autres étalons et/ou qui ne sont pas utilisés pour tous les types d'échanges au sein d'une

1 Gérin et al. 2001, p. 5. 
société donnée, dont la valeur n'est ni déterminée, ni garantie par un État, etc. et qui possèdent à la fois des fonctions économiques, socio-politiques et religieuses $[\ldots]^{2}$.

Se pose également le problème de l'estampille garantissant le poids et le titre. Outre le fait qu'on imagine difficilement une autorité souveraine estampiller des bœufs ou des coquillages, le métal lui-même a pu et peut encore être utilisé à la pesée, sans marque de contrôle d'aucune sorte ${ }^{3}$. L'idée du recours à la monnaie pour faciliter, ou tout le moins servir aux échanges, ne s'impose pas non plus comme une évidence.

S'interroger sur l'apparition de la monnaie frappée revient donc à se poser plusieurs questions simples, que l'on peut résumer de la manière suivante : Où et Quand ? Comment et pourquoi ${ }^{4}$ ?

L'étude des auteurs antiques ne s'avère pas d'une grande aide pour appréhender les débuts de la monnaie en Grèce (et d'une manière générale dans le monde égéen) : les Anciens considèrent l'apparition de la monnaie comme une évidence qu'ils replacent dans une réflexion éthique (et souvent non dénuée d'anachronismes) sur le rôle des grands législateurs 5 . Pourtant, l'origine géographique et chronologique de la monnaie frappée dans le monde méditerranéen semble aujourd'hui faire consensus, même si la question ne paraît pas tout à fait tranchée ${ }^{6}$. Sur ce sujet, les deux dépôts découverts en 1904-1905 lors des fouilles de l'Artémision d'Éphèse apparaissent essentiels ${ }^{7}$; leur datation a fait l'objet de trois propositions. D. G. Hogarth, directeur des fouilles entreprises par le British Museum, situait l'apparition du monnayage au VIII ${ }^{\mathrm{e}}$ siècle, mais B. V. Head - chargé d'étudier spécifiquement ces monnaies - ne remonta pas aussi haut, s'arrêtant au VII ${ }^{\mathrm{e}}$ siècle $^{8}$. Récemment, D. Williams a proposé de faire commencer

2 Grandjean 2001, p. 10. L'ensemble des actes de cette journée d'études fut publié dans la Revue Numismatique, 157 en 2001.

3 Sur l'utilisation, par exemple, des « monnaies » métalliques en Mésopotamie, voir Lambert 1963 ; Joannès 1994 ; Le Rider 2001, p. 1-40 ; Lion 2013.

4 Ces questions furent déjà posées, avec des réponses différentes, par Callataÿ 2001.

5 Picard 2001.

6 La question des origines de la monnaie est extrêmement complexe. Ce propos se place dans une vision résolument méditerranéo-centrée et exclut la naissance, indépendante, de la monnaie métallique en Chine à la fin du XII ${ }^{\mathrm{e}}$ siècle avant J.-C.

7 Les monnaies trouvées lors de ces fouilles ont été présentées par Robinson 1951, p. 156-158 et p. 166167. Ses conclusions ont été suivies par Weidauer 1975, p. 72-80 ; Karwiese 1991, p. 3-5 et Le Rider 2001, p. 59. Le reste du matériel accompagnant les monnaies a été publié par Jacobsthal 1951.

8 Head 1911, p. 644. 
ce monnayage aux alentours de $650^{\circ}$. Une chronologie moyenne a aussi été proposée par E. S. G. Robinson, qui datait ces monnaies d'une période postérieure à 630, suivi en cela par de nombreux auteurs, dont M. J. Price et I. Carradice. Ce dernier considérait même que le dépôt aurait été enfoui vers $600^{10}$. Une troisième chronologie, résolument plus basse, a été avancée par M. Vickers, pour qui le terminus ante quem des premières monnaies d'électrum doit être placé vers 520 avant J.-C. ${ }^{11}$

Les travaux d'A. Bammer ont montré que, même si la question reste ouverte ${ }^{12}$, la chronologie haute doit être définitivement abandonnée, le terminus ante quem devant être placé vers $560-550^{13}$. Les premières monnaies d'électrum signées et frappées le furent donc très probablement sous Alyatte(II). Mais d'après la doxa héritée des Anciens, la frappe des premières monnaies d'or et d'argent purs serait à attribuer à

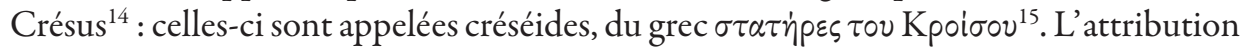
de ces frappes à Crésus reste cependant discutée. Hérodote et Xénophane de Colophon ont fait des Lydiens les premiers hommes à avoir frappé du numéraire d'or et d'argent, opinion largement partagée par les Grecs en raison de la très grande richesse de ce

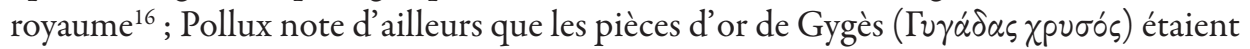
aussi renommées que les statères de Crésus ${ }^{17}$. Néanmoins, G. Le Rider a souligné la complexité du problème :

Que les premières monnaies d'électrum aient été frappées en Asie Mineure occidentale et dans les îles voisines ne fait aucun doute. Les lieux de trouvailles l'indiquent clairement. [...Mais] leur classement par atelier d'émission est beaucoup plus malaisé ${ }^{18}$.

9 Williams 1991-1993.

10 Carradice, Price 1988, p. 24-26. Voir également Kraay 1976, p. 21-22 ; Holloway 1984; Wallace 1987, p. 385 et Karwiese 1991, p. 22-23 et p. 27-28.

11 Vickers 1985 , p. 9-22.

12 Le Rider 2001, p. 67.

13 Bammer 1990 et 1991.

14 Hérodote, I, 94. La question reste néanmoins insoluble à la seule lecture des philosophes et historiens antiques car, comme l'a bien montré $\mathrm{O}$. Picard, ces derniers ne perçoivent pas la monnaie comme le résultat d'une évolution économique ou sociale, mais comme un invariant institutionnel des cités, issu la plupart du temps d'un législateur dont l'historicité peut être parfois - pour ne pas dire toujours - sujet à caution (voir Picard 2001).

15 Plutarque, OEuvres morales, 822 F-823A et Pollux, Onomasticon, III, 87 et IX, 84.

16 Hérodote, I, 93-94 et Xénophane, Onomasticon, IX, 83.

17 Pollux, Onomasticon, III, 87 : Eủdókıн

18 Le Rider 2001, p. 43. 
Les fouilles archéologiques et les études métrologiques révèlent toutefois que

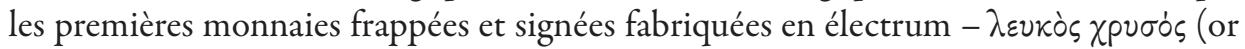
blanc) chez Hérodote - se répartissaient en quatre étalons différents : l'étalon « lydomilésien » (figure 1), l'étalon « phocaïque » (figure 2), l'étalon «samien lourd » (figure 3) et l'étalon « samien léger » (figure 4) ${ }^{19}$, sans que l'on puisse établir une éventuelle hiérarchie parmi les ateliers.
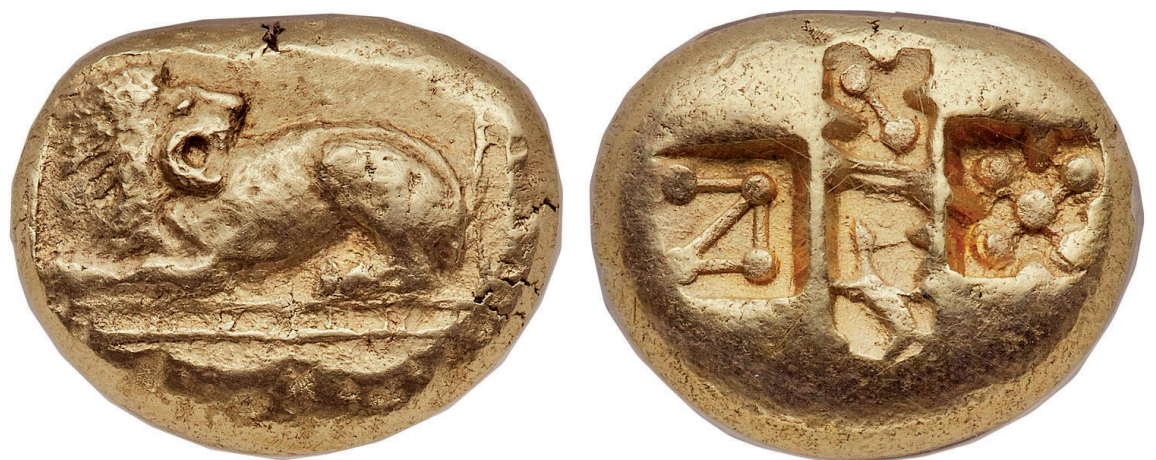

Figure 1 : Statère (électrum), Milet, vers 600-530 avant J.-C. (19 mm, 13,97 g) - Heritage World Coin - Auctions NYINC Signature Sale 3061, lot 32050 (07/01/2018).
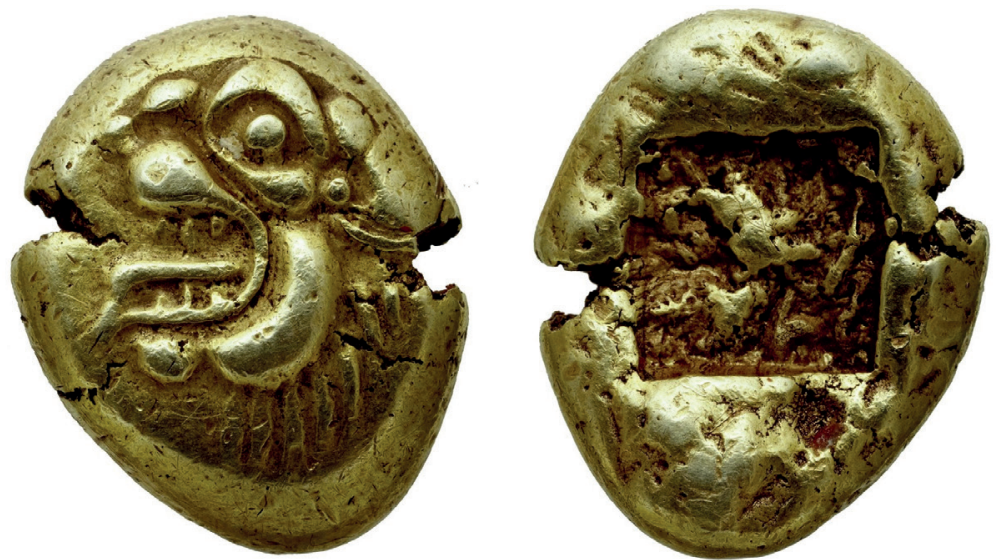

Figure 2 : Statère (électrum), Phocée, vers 550-500 avant J.-C. (22 mm, 16,06 g) - BnF - DMMA, Luynes 2655 (cliché P.-O. Hochard).

19 Sur les premiers monnayages d'électrum, les études fondamentales restent Robinson 1951, Robinson 1958 et Weidauer 1975. On pourra également se référer plus récemment à Ramage, Craddock 2000 et Bresson 2006. 

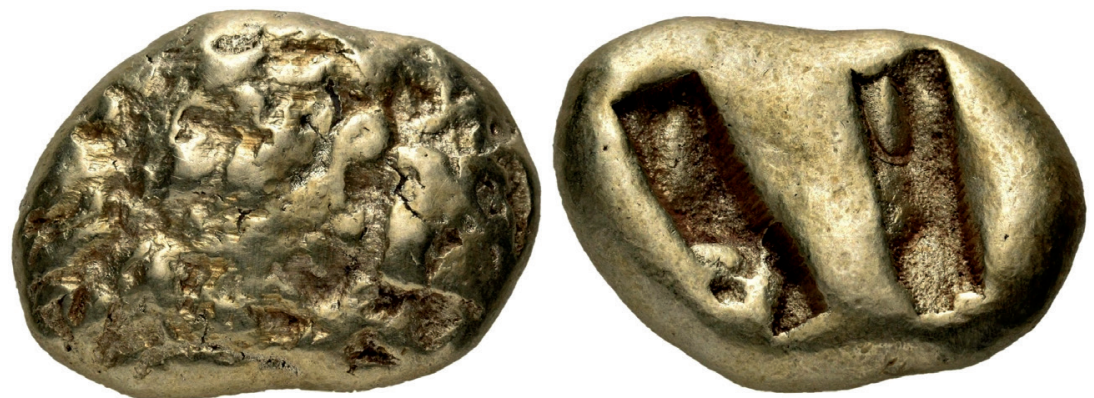

Figure 3 : Statère lourd (électrum), Samos, vers 600-570 avant J.-C. (23 mm, 17,27 g) - Nomos AG - Auction 15, lot 157 (22/10/2017).
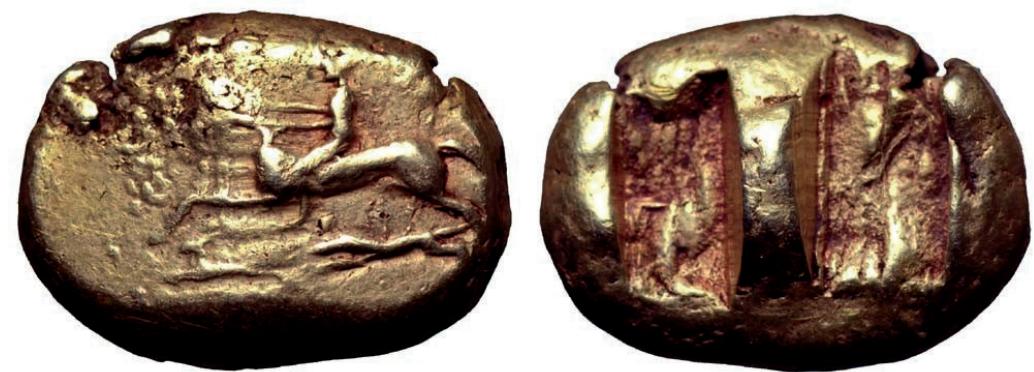

Figure 4 : Statère léger (électrum), Samos, vers 550-500 avant J.-C. (20 mm, 13,45 g) - Roma Numismatics Limited Auction 10, lot 427 (27/09/2015).

L. Weidauer a cependant identifié, en ce qui concerne l'ensemble des monnaies d'étalon lydo-milésien, trois ateliers dont l'existence est quasiment certaine: Sardes, Milet et Éphèse. Au $\mathrm{VI}^{\mathrm{e}}$ siècle, le monnayage sardien était le plus abondant d'Asie Mineure ${ }^{20}$, ce qui ne prouve nullement la paternité lydienne de l'invention de la monnaie même si, à la suite d'Hérodote ${ }^{21}$, l'historiographie traditionnelle a fait des Mermnades des précurseurs en la matière. La plupart des spécialistes admet que Crésus fut peut-être l'initiateur des premières monnaies d'or et d'argent mais ils attribuent

20 Le Rider 2001, p. 47. Pour un résumé des problématiques autour des premières monnaies d'électrum, Le Rider 2001, p. 41-47 ; Gérin et al. p. 41-43 ; Bresson 2008, p. 49-50. On peut attribuer aux rois de Lydie des monnaies d'électrum sans type avec la marque d'un poinçon au revers, les séries à la tête de lion à droite, aux deux têtes de lion, à la patte de lion, aux deux têtes de sangliers, à la protomé de lion à droite et aux protomés adossées de lion et de taureau.

21 Hérodote, I, 94, 1. 
cependant l'essentiel de la production aux Perses, après leur conquête de la Lydie aux alentours de 546. La frappe des créséides aurait cessé avec la réforme monétaire entreprise par Darius I ${ }^{\text {er22 }}$, qui les aurait définitivement remplacées par les dariques d'or et les sicles d'argent, de même étalon que les créséides légères. Les liaisons de revers entre les monnaies des deux métaux et leur étude stylistique donnent à penser que Sardes fut le seul atelier à émettre ces nouvelles monnaies ${ }^{23}$. Ainsi est-il maintenant couramment admis que les premières émissions de monnaies frappées eurent lieu en Asie Mineure occidentale, dans le royaume de Lydie, au $\mathrm{VI}^{\mathrm{e}}$ siècle avant notre ère.

Se pose alors la question beaucoup plus délicate du comment et du pourquoi ? Elle renvoie à d'autres interrogations, déjà formulées par J. Andreau ${ }^{24}$ :

- En Grèce comme en Asie Mineure, l'apparition de la monnaie frappée est-elle perçue comme une invention ou une innovation ${ }^{25}$ ?

- La monnaie frappée fut-elle originellement publique ou privée ?

- Quelle importance historique accorder à la monnaie frappée ?

- Comment expliquer le retard de certains États (cités, royaumes...) dans son adoption?

Plusieurs raisons ont été avancées pour expliquer l'apparition de la monnaie signée, frappée et de poids contrôlée ${ }^{26}$. Sous l'influence d'Aristote ${ }^{27}$, certains y ont vu une tentative de simplifier les échanges en évitant les opérations de pesée et de vérification ${ }^{28}$. Pourtant, les premières monnaies ne semblent pas avoir eu vocation à alimenter le grand commerce «international ». L'argument commercial ne tient pas, en effet, dès lors que l'on sait que, durant des millénaires, les Mésopotamiens recoururent aux simples lingots de métal. Si le système ne leur avait pas donné pleine satisfaction, il n'aurait probablement pas tenu aussi longtemps. De plus, C. M. Kraay a montré que

22 Concernant le débat de l'attribution des créséides au dernier Mermnade ou à Cyrus le Grand, voir Le Rider 2001, p. 101-121 pour un résumé des débats et de la bibliographie sur la question.

23 Gerin et al. 2001, p. 44.

24 Andreau 2011, p. 166.

25 J. Andreau pose la question de la manière suivante : « Est-ce qu'en Grèce en en Asie Mineure, avant cette apparition, il y avait des objets remplissant les trois fonctions de la monnaie ? ». Je reviendrai dans la suite du propos sur la question des trois fonctions.

26 Parmi les articles et écrits exposant les diverses explications, voir Holloway 1978; Le Rider 2001, p. 71-84.

27 Aristote, Politique, I, 9, 1257a.

28 Babelon 1897 ; Head 1911 ; Seltman 1955. 
les premières monnaies circulèrent peu hors de leur territoire d'émission ${ }^{29}$. Comme le souligne G. Le Rider :

elles ont pu simplifier certains échanges dans une région déterminée [...], mais il existe d'autres facteurs qui ont contribué au succès éclatant et durable qu’a connu la monnaie signée et frappée $e^{30}$.

L'apparition de la monnaie a aussi été attribuée à une volonté de faciliter les opérations comptables, hypothèse qui, cependant, ne fait pas l'unanimité. Cette idée, développée par R. M. Cook, fut reprise et étayée par C. M. Kraay ${ }^{31}$. L'hypothèse a suscité les réserves de Chr. Howgego et G. Le Rider ${ }^{32}$. Les pratiques mésopotamiennes offrent une nouvelle fois un contre-exemple, ou tout au moins un élément de modération. L'administration fiscale et financière des souverains assyro-babyloniens - et achéménides - fut sans doute au moins aussi complexe que celle des rois mermnades. L'usage des lingots ou du métal pesé et anonyme est cependant attesté, même de façon partielle, dans la rétribution des soldats ou des travailleurs $\mathrm{du}^{\mathrm{e}}$ siècle, aussi bien à Persépolis qu’à Éléphantine ${ }^{33}$. Pourtant, l'usage de la monnaie frappée et signée était déjà bien connu. Et G. Le Rider de conclure :

De même que l'explication « commerciale » des premières monnaies d'électrum n'a pas semblé complètement satisfaisante, de même l'explication «comptable », malgré son intérêt, ne paraît pas à elle seule donner la réponse au problème posé ${ }^{34}$.

Au début des années 1920, B. Laum avait développé l'idée que la monnaie avait été créée dans un contexte religieux, lorsque la nécessité de substituer un bien à un autre dans le cadre du culte et des sacrifices s'était faite sentir ${ }^{35}$. Largement décrié pour son approche excessive et l'outrance de sa démonstration, B. Laum ouvrit cependant la voie à une réflexion originale sur les origines de la monnaie frappée, éloignée des préoccupations économique et mercantile. Certains auteurs, tels Éd. Will et M. I. Finley ${ }^{36}$, ont en effet mis l'accent sur des considérations sociales ou politiques.

\footnotetext{
29 Kraay 1964. Voir également sur ce point Price 1983 ; Callataÿ 2001, p. 88-89.

30 Le Rider 2001, p. 74.

31 Cook 1958, p. 261 ; Kraay 1964, p. 88-91; Kraay 1976, p. 317-324.

32 Howgego 1995, p. 3-4; Le Rider 2001, p. 75-76.

33 Pour Persépolis, voir Naster 1970 ; concernant Éléphantine, voir Picard 1978, p. 19.

34 Le Rider 2001, p. 76.

35 Laum 1924. Cette idée rejoint celle de la dette de vie sur laquelle je reviendrai.

36 Will 1954; Will 1955 et Finley 1965.
} 
S'appuyant sur les travaux d'E. Laroche, Éd. Will rappelle que le terme vó $\mu \iota \sigma \mu \alpha$, dérivé de vóuos, s'inscrit en effet dans un ensemble sémantique où la notion d'éthique est omniprésente ${ }^{37}$. D'autres savants ont avancé l'idée que la monnaie n'aurait servi que de support idéologique, par le biais de l'iconographie et plus généralement des messages véhiculés par la monnaie frappée et signée. Dans son Traitésur la monnaie, J. M. Keynes émit l'hypothèse que les rois de Lydie ne cherchèrent rien d'autre, dans l'émission des premières monnaies d'électrum, que l'ostentation pure; M. I. Finley reprit cette idée, ajoutant :

Pride and patriotism constituted a serious and sufficient motive, far more than most historians or numismatists seem prepared to allow ${ }^{38}$.

Cette thèse trouva un certain écho chez C. M. Kraay et M. J. Price : ce dernier l'appliqua non à l'État mais à des particuliers qui auraient pu, selon lui, frapper les premières monnaies d'électrum pour affirmer leur autorité et leur prestige ${ }^{39}$. Deux ouvrages, paru à la fin des années 1990, reprirent à nouveaux frais cette approche anthropologique des origines de la monnaie. Pour S. von Reden, dont l'approche est résolument sociale, l'apparition de la monnaie répond à un besoin de justice ${ }^{40}$. La monnaie frappée serait donc le témoin (non unique par ailleurs) des acquis sociaux et démocratiques qui touchent progressivement les cités grecques à partir du VI ${ }^{\mathrm{e}}$ siècle. Les premières émissions monétaires, particulièrement à Athènes et Corinthe, auraient donc été le fait non des cités, mais d'institutions ou de riches particuliers soucieux de paix sociale : l'auteur en veut pour preuve la grande diversité iconographique des premières frappes ${ }^{41}$. Publié deux ans plus tard, l'ouvrage de L. Kurke approfondit encore la réflexion anthropologique : prenant la suite des analyses religieuse de Laum, éthique de Will et sociale de von Reden, l'auteur privilégie l'analyse politique. Elle insiste donc sur les oppositions entre une tradition élitiste et un mouvement populaire, en traquant dans les sources issues de la transmission manuscrite - et particulièrement Hérodote les traces d'une telle opposition dans le contexte d'émergence de la monnaie ${ }^{42}$. Ces ouvrages ont suscité des critiques nombreuses, malgré l'intérêt intellectuel des

\footnotetext{
37 Laroche 1949; Will 1954, p. 213.

38 Keynes 1930, p. 12 ; Finley 1965, p. 22.

39 Kraay 1964 et 1976, p. 317-324; Price 1983.

40 Von Reden 1995. Cette idée est également reprise dans von Reden 1997.

41 L'idée d'une origine privée de la monnaie frappée fut également soutenue par Price 1983.

42 La même hypothèse avait déjà été développée autour de la poésie par Morris 1987.
} 
idées développées ${ }^{43}$. Outre les critiques que l'on pourrait qualifier de techniques et «ponctuelles » (reconnaître, par exemple, dans l'iconographie des Wappenmünzen les blasons des grandes familles athéniennes n'est plus guère une idée défendue par les numismates), le principal reproche formulé envers ces deux livres fut de rester au niveau des généralités et de ne pas prendre en compte les difficultés concrètes de la mise en place des premières monnaies frappées. Comme le résume Fr. de Callataÿ :

je ne crois pas qu'il faille accueillir sans réserve l'explication alternative de la monnaie comme instrument de conquête sociale. Il s'agit là d'une fable ou, plus correctement d'un autre raccourci ${ }^{44}$.

D’une manière générale, l'approche anthropologique rappelée précédemment pose le problème de l'utilité réelle du recours à la monnaie frappée : une relecture attentive d'Éd. Will emporte l'adhésion sur le schéma général du raisonnement mais suscite la réserve lorsqu'on se demande si le recours à la monnaie stricto sensu était absolument nécessaire pour arriver à ce résultat.

Pour bon nombre d'historiens et de numismates, tous ces raisonnements, pourtant pertinents, auraient négligé l'essentiel : les aspects fiscaux. Cette idée avait déjà été évoquée par les spécialistes défendant le recours à la monnaie frappée pour procéder à des paiements standardisés ${ }^{45}$ ou pour valoriser un type de ressource ${ }^{46}$. C'est néanmoins G. Le Rider qui défendit le plus fortement l'idée d'une origine fiscale de la monnaie frappée et signée. Il insiste sur le fait que la recherche du profit a toujours existé dans les transactions commerciales et tout ce qui relève de la « chrématistique » selon Aristote, mais il relève trois points sur lequel l'État pouvait tirer profit de l'émission même d'une monnaie à son nom et à ses types, émissions dont il aurait conservé le monopole : l'écart entre valeur intrinsèque et valeur nominale (il estime qu'à Athènes au $\mathrm{V}^{\mathrm{e}}$ siècle, la valeur nominale d'une pièce était probablement supérieure de $5 \%$ à sa valeur intrinsèque ${ }^{47}$ ); le change imposé par l'État dans le cadre d'un système monétaire fermé - situation fréquente selon luii ${ }^{48}$; les manipulations monétaires à l'intérieur des frontières lorsque le besoin s'en faisait sentir. Alors que la théorie émise par G. Le Rider

\footnotetext{
43 Voir particulièrement sur ce point Callataÿ 2001.

44 Callatä̈ 2001, p. 88

45 Cook 1958 ; Kraay 1964 et 1976 ; Price 1983.

46 Wallace 1987 ; Kroll 1998; Descat 2001.

47 Le Rider 2001, p. 257-259.

48 Le Rider 2001, p. 80.
} 
a suscité l'adhésion d'un grand nombre de spécialistes de la monnaie ${ }^{49}$, cette hypothèse se heurte pourtant à la comparaison avec des États ne recourant pas à la monnaie frappée et signée. G. Le Rider souligne d'ailleurs lui-même, à la suite d'O. Picard, que les États mésopotamiens tirant d'énormes revenus de la terre n'avaient aucune raison de s'intéresser ou de développer cette nouvelle ressource fiscale ${ }^{50}$. C'est selon lui ce qui distingue d'ailleurs fondamentalement le monde égéen des empires moyen-orientaux $\mathrm{du} \mathrm{I}^{\mathrm{er}}$ millénaire :

La cité grecque, au contraire, avait des revenus fonciers très faibles et sa trésorerie était alimentée surtout par des taxes diverses, des amendes; elle était souvent à court de ressources et cherchait sans cesse des moyens d'augmenter ses recettes. Crésus lui-même, ajoute O. Picard, ne disposait pas de possibilités aussi vastes que les rois babyloniens ou mèdes ${ }^{51}$.

L'hypothèse de G. Le Rider est fortement discutée par J. H. Kroll, qui y oppose le cas des poids athéniens du $\mathrm{VI}^{\mathrm{e}}$ siècle ${ }^{52}$. A. Orléan invite quant à lui à beaucoup de prudence face à cette hypothèse, insistant sur l'aspect ponctuel de ce profit (en effet, l'État sera in fine lui-même payé avec ces monnaies dont la valeur nominale et la valeur réelle different, annulant le gain provoqué par l'émission de ces monnaies) et sur l'existence concomitante de monnaies dont la valeur réelle et la valeur nominale sont exactement semblables (l'auteur prend l'exemple des dariques d'or) ${ }^{53}$. Une nouvelle fois, bien que séduisante et stimulante, une telle hypothèse se heurte aux difficultés concrètes de la réalité.

Il reste donc difficile d'attribuer l'apparition de la monnaie à un facteur unique, et la persistance, après le $\mathrm{VI}^{\mathrm{e}}$ siècle, de cités ne frappant pas monnaie - telles Sparte ou Rome, qui n'inaugurèrent un monnayage propre qu' au $\mathrm{III}^{\mathrm{e}}$ siècle avant J.-C. - tend à montrer que les frappes n'étaient pas destinées à alimenter une circulation régulière et ne relevaient pas d'une quelconque politique monétaire, du reste anachronique pour les sociétés antiques ${ }^{54}$.

49 On peut, pour s'en convaincre, se reporter aux notes additionnelles de Bresson 2001, p. 68 et surtout Callataÿ 2001, p. 92.

50 Picard 1978 , p. 20.

51 Le Rider 2001, p. 82-83.

52 Kroll 2001.

53 Orléan 2002.

54 Bresson 2001 ; Callataÿ 1997 et 2005 ; Bresson 2008. 
Si les historiens de la monnaie antique se sont très rapidement posés la question $\mathrm{du}$ « où et quand ? » avec un certain succès, puis du « comment et pourquoi ? » avec plus de difficultés - et sans avoir, semble-t-il, épuisé le sujet-la question du « quoi ? » ne paraît pas (etj' insiste sur ce mot) avoir déchaînéles passions. Fortement influencés par les propos d'Aristote, les spécialistes de la monnaie - qu'ils soient historiens, économistes ou sociologues - ont souvent abordé la question de la monnaie frappée sous l'angle de la simplification des échanges. Cette dernière est donc la plupart du temps définie, de façon souvent implicite, par ses trois fonctions : intermédiaire des échanges, unité de compte et réserve de valeur. Pour reprendre une expression de J. A. Schoonheyt, il y aurait ainsi la monnaie - c'est-à-dire la monnaie frappée - et le reste (pré, paléo, proto, paramonnaie $)^{55}$. Cette idée est celle défendue par la théorie économique classique ou néo-classique (que j'appellerai ici la théorie orthodoxe) et la fameuse « fable du troc $\gg^{56}$, dans laquelle la monnaie ne fut inventée que pour surmonter les difficultés liées à ce dernier. De ce fait, la monnaie devient un objet tout naturellement économique, neutre et par là-même sans existence significative ${ }^{57}$. Pourtant, dans l'arbre de décision sur les moyens d'échange qu'il dresse, J. A. Schoonheyt isole 24 catégories de moyen d'échange, la monnaie frappée et signée occupant la $24^{\mathrm{e}} \mathrm{case}^{58}$. Et l'auteur de conclure :

Cette arborescence rend donc compte des moyens d'échange sans limitation dans le temps ni dans l'espace : ainsi le dollar américain est imposé et légal aux États-Unis, mais il est accepté non légal dans d'autres pays du monde et sert également d'étalon de valeur dans les grandes transactions internationales ${ }^{59}$.

Puisque la monnaie frappée - qui se définit alors comme un moyen d'échange imposé et légal s'inscrivant dans un système pondéral, de forme définie et dont la valeur intrinsèque est garantie par une marque - peut cohabiter avec d'autres moyens d'échange dans un même espace-temps, on peut donc considérer, à l'instar de R. Descat, que le passage à la monnaie frappée est indiscutablement

un phénomène complexe dont la compréhension ne se réduit pas aux premières émissions qui ont certainement des fonctions en partie différentes des monnayages suivants ${ }^{60}$.

\footnotetext{
55 Schoonheyt 2001, p. 33 dans le résumé.

56 Sur l'origine et le succès de cette fable, voir Servet 1994.

57 Sur ce point, voir Aglietta, Orléan 1998, p. 16.

58 Schoonheyt 2001 (voir dans ce volume l'introduction p. 11).

59 Schoonheyt 2001, p. 36.

60 Descat 2001, p. 77.
} 
C'est ainsi qu'un courant hétérodoxe, composé d'économistes, mais aussi d'historiens et d'anthropologues, développa une approche radicalement différente de la monnaie, conçue comme un élément du « tout social », et donc fondamentalement non-neutre. Théorisée dans La monnaie souveraine, parue en $1998^{61}$, cette conception socialement totalisante part du principe que la monnaie n'est pas ce qu'elle fait ${ }^{62}$, et repose sur un trépied dont le premier fondement est la dette. Les auteurs exposent le fait que les relations entre individus sont fondées, quelles que soient les sociétés, sur le principe d'une dette, privée ou publique. Parmi toutes ces dettes, l'une en particulier prend tout son sens pour expliquer ce qu' est la monnaie et comprendre son origine : la dette de vie. Chaque individu étant débiteur du « capital-vie » qu'il reçoit et créditeur $\mathrm{du}$ « capital-vie » qu'il transmet, la monnaie permet d'entretenir le lien social entre les différentes générations, en permettant à chacun de s'acquitter de ses dettes, qu'elles soient remboursables au cours de sa vie ou perpétuelles et non remboursables par le débiteur $^{63}$. Compte tenu de la mortalité de ses membres, une société, quelle qu'elle soit, doit trouver le moyen d'assurer sa pérennité, en transmettant à travers les générations son « capital-vie » et donc sa dette de vie. Seul l'État peut alors assurer cette continuité du groupe social par-delà le cycle générationnel. La souveraineté apparaît donc comme le deuxième pilier du trépied soutenant la monnaie comme élément du tout social. À partir de là, le dernier soutien de ce trépied est la confiance, que le groupe social met dans l'État pour garantir la souveraineté, et donc la soutenabilité de la dette primordiale. Selon les auteurs de La monnaie souveraine, ce recours à la confiance provoque deux conséquences fondamentales: d'une part, la monnaie, y compris la monnaie frappée en métal précieux, ne peut qu'être fiduciaire ${ }^{64}$; d'autre part, cette idée de confiance ne saurait se réduire à la simple notion de crédibilité. Ainsi la confiance prendrait-elle trois formes : méthodique (ou déméfiance), hiérarchique et éthique.

Ainsi la confiance éthique est à la confiance hiérarchique ce que la légitimité est à la légalité et ce que l'autorité symbolique est au pouvoir politique. Au total, pour être lien de confiance, la monnaie requiert le jeu combiné de ces trois formes. Selon cette conception, la souveraineté tient un rôle central dans la confiance car, si la souveraineté est légitime,

\footnotetext{
61 Voir en particulier Aglietta, Orléan 1998, p. 9-31.

62 Expression empruntée à Théret 2008a, p. 39.

63 Rospabé 1995 ; Hénaff 2002.

64 Cette idée fut déjà développée pour les monnaies d’or et d'argent par Simiand 1934. Voir aussi Aglietta, Orléan 2002; Théret 2008a, p. 265-303.
} 
la confiance dans la monnaie est assurée, la confiance méthodique étant garantie par la confiance hiérarchique et celle-ci par la confiance éthique ${ }^{65}$.

Cet ouvrage n'a pas manqué de susciter la critique. Certains ont reproché aux auteurs leur approche trop théorique pour les sociétés modernes et ont contesté la définition tripartite faite de la confiance ${ }^{66}$; d'autres ont reproché à La monnaie souveraine une vision anhistorique de la monnaie, négligeant les discontinuités, ou encore l'imprécision de certaines définitions, notamment celle de souveraineté ${ }^{67}$. Les auteurs de La monnaie souveraine étaient cependant partiellement conscients des limites de leur propre travail. Ainsi M. Aglietta et A. Orléan profitèrent de la réflexion menée au cours de l'élaboration de La Monnaie souveraine pour reprendre et grandement réactualiser La violence de la monnaie (1982) et en faire, selon leurs propres mots, un ouvrage totalement nouveau, avec La Monnaie entre violence et confiance (2002). De nombreux contributeurs de La monnaie souveraine continuèrent également la réflexion entamée sur la question de la monnaie élément du « tout social », seuls ou de manière collective ${ }^{68}$. L'une des limites de La monnaie souveraine est d'ailleurs relevée par B. Théret, pourtant partie prenant de la rédaction de l'ouvrage de 1998, dans La monnaie dévoilée par ses crises:

La monnaie souveraine, il faut le reconnaître, a eu du mal à rompre clairement et explicitement avec une approche positiviste de la monnaie saisie à partir de ses «fonctions ». En cela, elle ne se distingue pas vraiment des autres théories institutionnalistes et symboliques qui, confrontées à la conception orthodoxe de la monnaie comme moyen d'échange marchand et à la vulgate d'une monnaie définie par les trois (ou quatre) fonctions d'étalon de valeur, de moyen de circulation (éventuellement séparé en moyen d'échange et moyen de paiement unilatéral) et de réserve de valeur, se limitent en général à poser trois restrictions : la fonction d'unité de compte est une fonction première logiquement et historiquement ; la fonction de moyen de paiement est également une fonction de base et englobe celle de moyen d'échange, et non l'inverse; la fonction de réserve n'est pas spécifiquement monétaire. Cela constitue certes un progrès mais, en réduisant la monnaie à ses fonctions d'unité de compte et de moyen de paiement, on continue de la définir par rapport à un extérieur à elle-même et non pas à partir de sa « nature » de relation sociale spécifique, créatrice de son propre espace social ${ }^{69}$.

65 Théret 2008a, p. 25. Sur la distinction entre légitimité et légalité, voir Bouyssou, Hochard 2016.

66 Grenier 2000.

67 Piron 2002 ; Hénaff 2002, p. 403-412; Caillé 2002.

68

Théret 2008a et 2008b ; Servet 2012.

69

Théret 2008a, p. 39. 
Plutôt que d'envisager la monnaie selon ses fonctions, et donc la définir par ce qu'elle fait, B. Théret propose de définir la monnaie par ses formes et ses états, donc ce qu' elle est. Il distingue ainsi l'état incorporé de la monnaie (où la monnaie est étalon de valeur et de confiance), l'état objectivé (regroupant les instruments monétaires servant de moyens de paiements) et l'état institutionnalisé (c'est-à-dire les règles unifiant un espace monétaire régi par un système de compte et constituant une communauté de paiement $)^{70}$. Comme pour La monnaie souveraine, La monnaie dévoilée par ses crises n'a pas manqué de susciter des débats - finalement de même nature que ceux déjà provoqués par La monnaie souveraine - entre partisans et opposants d'une conception de la monnaie comme élément d'un « tout social ${ }^{71}$. Le sujet est donc loin d'être épuisé, et le débat loin d'être clos.

La monnaie frappée est donc incontestablement une innovation monétaire, mais certainement pas une invention, encore moins une révolution. Le principal enjeu pour les historiens de la monnaie, surtout ceux s'intéressant aux origines de la monnaie stricto sensu, n'est donc pas tant dans le où et le quand, le comment et le pourquoi (nonobstant l'intérêt de ces questions) : l'enjeu est dans le quoi. S'interroger sur l'apparition de la monnaie frappée revient in fine à s'interroger sur l'apparition d'un nouveau moyen d'échange, et donc à se placer dans une évolution, comme le soulignait déjà R. Descat ${ }^{72}$. Questionner la naissance de la monnaie frappée, c’est questionner ce qu' est la monnaie en général, questionner le phénomène monétaire. Malgré son apparente universalité, ce dernier n'a rien d'un objet anhistorique, et ne saurait se réduire ou être réduit à sa seule dimension économique ou anthropologique. Il convient donc de replacer l'étude du phénomène monétaire (que la monnaie soit frappée ou pas) dans un temps long, et dans une démarche pluri et transdisciplinaire.

\section{Bibliographie}

Aglietta M., Orléan A. (2002), La monnaie entre violence et confiance, Paris.

Aglietta M., Orléan A. (dir.) (1998), La monnaie souveraine, Paris.

Aglietta M., Orléan A. (1982), La violence de la monnaie, Paris.

\footnotetext{
70 Théret 2008a, p. 42-53.

71 Voir par exemple Boyer 2008 ; Baubeau 2010.

72 Descat 2001.
} 
Andreau J. (2001), «La monnaie et les "monnaies frappées" conclusions de la table ronde du 15 janvier 2000 », Revue Numismatique, 157, p. 163-172.

Babelon E. (1897), Les origines de la monnaie considérée au point de vue économique et historique, Paris.

Bammer A. (1991), « Les sanctuaires des viII ${ }^{\mathrm{e}}$ et VII ${ }^{\mathrm{e}}$ siècles à l'Artémision d'Éphèse », RA, fasc. 1, p. 6383.

Bammer A. (1990), «A Peripteros of the Geometric Period in the Artemisium of Ephesus », AS, 40, p. $137-160$.

Baubeau P. (2010), « Bruno Théret (éd.), La monnaie dévoilée par ses crises, volume 1 : Crises monétaire d'bier et d'aujourd'hui; volume 2 : Crises monétaires en Russie et en Allemagne au XX' siècle », Revue d'histoire moderne et contemporaine, 57/3, p. 210-212.

Bellinger A. R. (1968), « Electrum Coins from Gordion », dans C. M. Kraay, G. K. Jenkins, Essays in Greek coinage presented to Stanley Robinson, Oxford, p. 10-15.

Bouyssou G., Hochard P.-O. (2016), «Tyrannis, basileia, imperium, les composantes militaires des légitimités politiques dans le monde gréco-romain ( $\mathrm{v}^{\mathrm{e}}$ siècle avant-IV $\mathrm{v}^{\mathrm{e}}$ siècle après J.-C.) », HIMA, 4, p. 5-16.

Boyer R. (2008), « Bruno Théret (dir.), La monnaie dévoilée par ses crises, Paris, 2007 », Revue de la régulation, $3 / 4 \mid 2^{\mathrm{e}}$ semestre [en ligne : http://regulation.revues.org/4813].

Bresson A. (2008), L'économie de la Grèce des cités, II, Les espaces de l'échange, Paris.

Bresson A. (2007), L'économie de la Grèce des cités, I, Les structures et la production, Paris.

Bresson A. (2006), « The Origin of Lydian and Greek Coinage : Cost and Quantity », Historical Research, 5, p. 149-165 [publié en traduction chinoise].

Bresson A. (2001), « Monnayages et sociétés dans les mondes antiques », Revue Numismatique, 157, p. 51-68.

Caillé A. (2002), « Quelle dette de vie ?», L’Homme, 162, p. 243-254.

Callatä̈ Fr. de (2005) : «L'étude des monnaies et l'histoire économique du monde grec », BAB, 7-12, p. 539-558.

Callataÿ Fr. de (2001), « Sur les origines de la monnaie stricto sensu (nomisma). À propos de deux livres récents (S. von Reden et L. Kurke) », Revue Numismatique, 157, p. 83-93.

Callataÿ Fr. de (1997), Histoire des guerres mithridatiques vue par les monnaies, Louvain-La-Neuve.

Carradice I., Price M. J. (1988), Coinage in the Greek World, Londres.

Crawford M. (1970), « Money and Exchange in the Roman World », JRS, 60, p. 40-48.

Cook R. M. (1958), « Speculations on the Origin of Coinage », Historia, 7, p. 257-262.

Descat R. (2001), « Monnaies multiples et monnaie frappée en Grèce archaïque », Revue Numismatique, 157, p. $69-81$. 
Finley M. I. (1987), « Max Weber et la cité grecque », Sur l'Histoire Ancienne. La matière, la forme et la méthode, Paris.

Finley M. I. (1965), Deuxième conférence internationale d'histoire économique, Aix-en-Provence, Paris.

Gérin D. et al. (2001), La monnaie grecque, Paris.

Grandjean C. (2001), « Introduction », Revue Numismatique, 157, p. 9-13.

Grandjean C., Moustaka A. (éd.) (2013), Aux origines de la monnaie fiduciaire. Traditions métallurgiques et innovations numismatiques, Bordeaux.

Grenier J.-Y. (2000), « Penser la monnaie autrement », Annales HSS, 6, p. 1335-1342.

Haudry J. (2002), Juno Moneta, Aux sources de la monnaie, Milan.

Head B. V. (1911), Historia Numorum: a Manual of Greek Numismatics, Amsterdam.

Hénaff M. (2002), Le prix de la vérité. Le don, l’argent, la philosophie, Paris.

Holloway R. R. (1984), « The Date of the First Greek Coins: some Arguments from Style and Hoards », $R B N, 130$, p. 5-18.

Holloway R. R. (1978), « La ricerca attuale sull'origine della moneta », RIN, 80, p. 7-14.

Howgego C. J. (1995), Ancient history from Coins, Londres-New York.

Howgego C. J. (1990), « Why did Ancient States Strike Coins? », NC, 150, p. 1-25.

Jacobsthal P. (1951), « The Date of the Ephesian Foundation Deposit », JHS, 71, p. 85-95.

Joannès Fr. (1994), « Métaux précieux et moyens de paiement en Babylonie achéménide et hellénistique », Transeuphratène, 8, p. 137-144.

Karwiese S. (1991), « The Artemision Coin Hoard and the First Coins of Ephesus », RBN, 137, p. 1-28.

Keynes J. M. (1930), Traité sur la monnaie, Londres.

Kraay C. M. (1976), Archaic and Classical Greek Coins, Berkeley.

Kraay C. M. (1964), « Hoards, Small Change and the Origin of the Coinage », JHS, 84, p. 76-91.

Kroll J.H. (2001), « La naissance de la monnaie. Pratiques monétaires de l'Orient ancien [Georges Le Rider] », RSN, 80, p. 199-206.

Kroll J. H. (1998), « Silver in Solon' Laws » dans R. Ashton, S. Hurter (éds), Studies in Greek Numismatics in Memory of Martin Jessop Price, Londres, p. 225-232.

Kurke L. (1999), Coins, Bodies, Games and Gold. The Politics of Meaning in Archaic Greece, Princeton.

Le Rider G. (2001), La naissance de la monnaie. Pratiques monétaires de l'Orient ancien, Paris.

Lambert M. (1963), « L'usage de l'argent-métal à Lagash au temps de la III dynastie d'Ur », Revue d'assyriologie et d'archéologie orientale, 57, p. 79-92.

Laroche E. (1949), Histoire de la racine NEM- en grec ancien, Paris. 
Laum B. (1924), Heiliges Geld, eine historische Untersuchung über den sakralen Ursprung des Geldes, Tübingen.

Lion B. (2013), «Le bronze et ses usages économiques en Mésopotamie: le cas de Nuzi (xiv ${ }^{\mathrm{e}}$. a.C.) », dans C. Grandjean, A. Moustaka (éds) Aux origines de la monnaie fiduciaire. Traditions métallurgiques et innovations numismatiques, Bordeaux, p. 129-143.

Moreau J. (1969), « Aristote et la monnaie », REG, 82, fasc. 391-392, p. 349-364.

Morris I. (1987), Burial and Ancient Society: the Rise of the Greek City-State, Cambridge.

Naster P. (1970), « Were the Labourers of Persepolis Paid by Means of Coined Money? », AncSoc, 1, p. 129-134.

Orléan A. (2002), « Georges Le Rider. La naissance de la monnaie. Pratiques monétaires de l'Orient ancien $\gg$, Annales HSS, 57, p. 668-671.

Picard O. (2001), « Les philosophes grecs et la monnaie », Revue Numismatique, 157, p. 95-103.

Picard O. (1978), « Les origines du monnayage en Grèce », L'Histoire, n 6, p. 16-19.

Piron S. (2002), « La dette de Panurge », L'Homme, 162, p. 255-270.

Price M. J (1983), « Thoughts on the Beginning of Coinage », dans C. N. L. Brooke et al. (éd.), Studies in numismatics presented to Philip Grierson, Cambridge-New York, p. 1-10.

Ramage A., Craddock P. (éds) (2000), King Croesus' Gold: Excavations at Sardis and the History of Gold Refining, Mass.-Londres.

Robinson E. S. G. (1958), « Some Electrum and Gold Greek Coins », Centennial volume of the ANS, p. 585-594.

Robinson E. S. G. (1951), « The Coins from the Artemision Reconsidered », JHS, 71, p. 156-167.

Rospabé P. (1995), La dette de vie. Aux origines de la monnaie sauvage, Paris.

Schooneheyt J. A. (2001), « L'arbre de décision sur les moyens d'échange », Revue Numismatique, 157, p. 33-36.

Seltmann Ch. T. (1955 [1933]), Greek Coins. A History of Metallic Currendy and Coinage down to the Fall of the Hellenistic Kingdoms, Londres.

Servet J.-M. (2012), Les Monnaies du lien, Lyon.

Servet J.-M. (1994), « La fable du troc », Dix-huitième Siècle, 26, p. 103-115.

Simiand F. (1934), « La monnaie réalité sociale », Les Annales sociologiques, série D (1), p. 1-58.

Théret B. (dir.) (2008a), La monnaie dévoiléepar ses crises, volume I, Crisesmonétaires d'bier et d'aujourd'hui, Paris.

Théret B. (dir.) (2008b), La monnaie dévoilée par ses crises, volume II, Crises monétaires en Russie et en Allemagne au $X X^{e}$ siècle, Paris.

Von Reden S. (1997), « Money, Law and Exchange », JHS, 117, p. 154-176. 
Von Reden S. (1995), Exchange in Ancient Greece, Londres.

Vickers M. (1985), « Early Greek Coinage, a Reassessment », NC, 145, p. 1-44.

Wallace R. W. (1987), « The Origin of Electrum Coinage », AJA, 91, p. 385-397.

Weidauer L. (1975), Probleme der frühen Elektronprägung, Fribourg.

Will Éd. (1955), « Réflexions et hypothèses sur les origines du monnayage », Revue Numismatique, 17, p. $5-22$.

Will Éd. (1954), « De l'aspect éthique des origines grecques de la monnaie », $R H, 212$, p. 209-231.

Williams D. (1991-1993), « The Pot Hoard from the Archaic Artemision of Ephesus », BICS, 38, p. 98103. 